\title{
Trif-dependent induction of Th17 immunity by lung dendritic cells
}

\author{
BJ Hsia ${ }^{1,2}$, GS Whitehead ${ }^{1,2}$, SY Thomas ${ }^{1}$, K Nakano ${ }^{1}$, KM Gowdy ${ }^{1}$, JJ Aloor ${ }^{1}$, H Nakano ${ }^{1}$ and DN Cook ${ }^{1}$
}

\begin{abstract}
Allergic asthma is thought to stem largely from maladaptive Thelper 2 (Th2) responses to inhaled allergens, which in turn lead to airway eosinophilia and airway hyperresponsiveness (AHR). However, many individuals with asthma have airway inflammation that is predominantly neutrophilic and resistant to treatment with inhaled glucocorticoids. An improved understanding of the molecular basis of this form of asthma might lead to improved strategies for its treatment. Here, we identify novel roles of the adaptor protein, TRIF (TIR-domain-containing adapter-inducing interferon- $\beta$ ), in neutrophilic responses to inhaled allergens. In different mouse models of asthma, Trif-deficient animals had marked reductions in interleukin (IL)-17, airway neutrophils, and AHR compared with wild-type (WT) mice, whereas airway eosinophils were generally similar in these two strains. Compared with lung dendritic cells (DCs) from WT mice, lung DCs from Trif-deficient mice displayed impaired lipopolysaccharide (LPS)-induced migration to regional lymph nodes, lower levels of the costimulatory molecule, CD40, and produced smaller amounts of the Thelper 17 (Th17)-promoting cytokines, IL-6, and IL-1 $\beta$. When cultured with allergen-specific, naive T cells, Trif-deficient lung DCs stimulated robust Th2 cell differentiation but very weak Th1 and Th17 cell differentiation. Together, these findings reveal a TRIF-CD40-Th17 axis in the development of IL-17-associated neutrophilic asthma.
\end{abstract}

\section{INTRODUCTION}

Asthma is a chronic disease of the airways characterized by inflammation, airway constriction, and airway hyperresponsiveness (AHR). ${ }^{1}$ Eosinophils are present in the airways of most humans with asthma, ${ }^{2}$ and symptoms in these individuals are generally improved by inhaled corticosteroids, the standard treatment for mild to moderate asthma. ${ }^{1}$ The accumulation of eosinophils in the airway is largely dependent on the actions of interleukin (IL)-5, which is produced by T helper 2 (Th2) cells. Th2 cells also produce IL-4 and IL-13, which promote the generation of immunoglobulin $\mathrm{E}$ and mucus production, respectively. ${ }^{3}$ Despite the well-established role of Th2 cells and eosinophils in asthma, many individuals have non-eosinophilic forms of the disease that are resistant to inhaled corticosteroids. $^{4-6}$ In some individuals, neutrophils are the major inflammatory cell type in the airway, and their increased number is associated with reduced lung function and unresponsiveness to inhaled steroids. ${ }^{7-9}$ The presence of both neutrophils and eosinophils in the airway is associated with particularly low lung function and high utilization of healthcare resources. ${ }^{10}$ Patients with neutrophilic forms of asthma represent an unmet clinical need, and it is critical to gain an improved understanding of the molecular basis of their disease. Several studies suggest that the actions of T helper 17 (Th17) cells, which produce the cytokines IL-17A, IL-17F, and IL-22, contribute to neutrophil recruitment and AHR. ${ }^{11-14}$ Th17 cytokines do not recruit neutrophils directly but trigger airway epithelial cells to secrete neutrophil-attracting chemokines, such as IL-8 in humans and CXCL5 in mice. ${ }^{15}$ In addition, IL-17 can also act directly on smooth muscle to strengthen its contraction. ${ }^{16}$ Intriguingly, Th17 cells are steroid-resistant, ${ }^{17}$ suggesting a possible explanation for the steroid resistance seen with neutrophilic asthma.

A large body of evidence has shown that lipopolysaccharide (LPS), a major component of the outer cell wall of Gramnegative bacteria, is a potent activator of Th17 responses, largely through its actions on dendritic cells (DCs). ${ }^{18-21}$ This is also true in the airway, where inhaled LPS promotes very strong

\footnotetext{
${ }^{1}$ Laboratory of Respiratory Biology, National Institute of Environmental Health Sciences, National Institutes of Health, Research Triangle Park, North Carolina, USA. Correspondence: DN Cook (cookd@niehs.nih.gov)

${ }^{2}$ These authors contributed equally to this work.
} 
Th17 responses to co-inhaled ovalbumin (OVA). ${ }^{22}$ Furthermore, when common house dust extracts (HDEs) containing LPS are used as an adjuvant, mice lacking toll-like receptor 4 (TLR4) develop weak Th17 responses, even though their Th2 responses remain strong. ${ }^{23}$ Together, these data suggest that LPS in the environment might trigger Th17 responses to inhaled allergens and, in turn, lead to a neutrophilic asthma. In support of this, some studies have shown that household levels of LPS are associated with an increased prevalence of asthma, ${ }^{24,25}$ although in farming communities exposure to microbial products can be protective. ${ }^{26}$

Myeloid differentiation primary response gene 88 (MyD88) is a proximal adaptor protein utilized by all known TLRs with the exception of TLR3, which uses a different adaptor, TIRdomain-containing adapter-inducing interferon- $\beta$ (TRIF). ${ }^{27}$ TLR4 appears to be unique among TLRs in its ability to signal through both MyD88 and TRIF. A recent study of secreted proteins by LPS-stimulated macrophages revealed that, depending on the protein in question, MyD88 and TRIF can have redundant, synergistic, or unique functions. ${ }^{28} \mathrm{We}$ reasoned that the potency of LPS as a Th17-inducing adjuvant might stem from the ability of TLR4 to signal through two different signaling pathways that are each required for robust Th17 development. MyD88 is required for the development of Th2 responses and asthma-like features in mice following LPS-mediated allergic sensitization through the airway; ${ }^{29}$ however, a role for TRIF in the development of Th2 or Th17 responses to inhaled allergens has not been reported. In the present study, we observed that TRIF is required for the development of Th17-mediated neutrophilia, but not for Th2-mediated eosinophilia. In agreement with this observation, DCs prepared from the lungs of $\mathrm{Trif}^{-1}$ mice have a severely compromised ability to initiate Th17 responses but are able to stimulate Th2 differentiation. Thus, TRIF has a unique and previously unrecognized role in lung DCs to direct Th17 responses to inhaled allergens.

\section{RESULTS}

\section{Requirement of TRIF for airway neutrophilia in mouse} models of allergic asthma

Sterile HDEs represent an environmental source of adjuvants and allergens that humans inhale on an everyday basis. HDEs activate DCs in vitro ${ }^{30}$ and elicit asthma-like features when instilled into the airways of mice. ${ }^{23,31}$ The adjuvant activity of HDEs also promotes allergic sensitization to otherwise innocuous experimental allergens, such as highly purified OVA, and upon subsequent challenge with this same protein, mice develop asthma-like features including airway eosinophilia and neutrophilia. ${ }^{23,32-34}$ Using this model, we recently found that the neutrophil component of airway inflammation, but not the eosinophilic component, is dependent on TLR4. ${ }^{35}$ To investigate potential roles of TRIF in HDE-mediated allergic sensitization, we instilled airways of wild-type (WT) and $\mathrm{Trif}^{-1-}$ mice with OVA alone, or OVA mixed with an HDE containing the equivalent of $4 \mathrm{ng}$ LPS per mouse (HDE-1). Asthma-like responses were then elicited in these mice by a challenge with aerosolized OVA (Figure 1a). C57BL/6J mice receiving OVA alone had very few inflammatory cells in the bronchoalveolar lavage (BAL) after challenge (Figure 1b), in agreement with previous reports that an adjuvant is required for effective allergic sensitization. ${ }^{23,36}$ By contrast, $\mathrm{C} 57 \mathrm{BL} / 6 \mathrm{~J}$ mice sensitized to OVA using HDE-1 as an adjuvant (HDE/OVA) had many eosinophils and neutrophils in the airway following OVA challenge. Age-matched $\mathrm{Trif}^{-1}$ mice sensitized with HDE/ OVA and challenged with OVA also had many airway eosinophils but had markedly fewer airway neutrophils than did WT mice. Thus, Trif is required in this model for neutrophilic responses to allergen challenge. Mice sensitized with HDE-1 alone also developed neutrophilia and eosinophilia, likely in response to allergens present in the extracts. Neutrophilic responses were significantly lower and eosinophilic responses correspondingly higher in the Trif ${ }^{-1}$ mice compared with WT mice. The requirement of Trif for neutrophil recruitment in this model was not unique to HDE-1 because similar findings were observed when a different HDE, with a much higher concentration of LPS, 50 ng per mouse (HDE-2), was used to sensitize mice to OVA (Supplementary Figure S1b online).

TRIF is required for Th17 responses to inhaled allergen The reduced neutrophil recruitment to the airways of Trif $^{-/}$ mice might have been because of an intrinsic defect in the ability of these cells to migrate to the lung, or to perturbation of a pathway upstream of this migration event. To address the first possibility, we instilled the neutrophil-recruiting chemokine, CXCL5, directly into the airways of WT and Trif $^{-1-}$ mice and assessed neutrophil accumulation $4 \mathrm{~h}$ later. WT and Trif $^{-/}$ mice had similar numbers of neutrophils in BAL fluid (Figure 1c), ruling out the possibility that Trif expression in these cells is required for their chemotactic responses to chemokines.

We next investigated pathways associated with neutrophil recruitment during adaptive immune responses. Previous studies have shown that airway neutrophilia in mouse models of asthma is closely associated with pulmonary production of IL-17. ${ }^{11-14}$ We therefore measured amounts of this cytokine in the lungs of OVA-challenged mice that had previously been sensitized to OVA using either HDE-1 or HDE-2. In each of these experiments, lungs of OVA-challenged Trif $^{-/}$mice had significantly less IL-17 than did lungs of similarly treated WT mice (Figure 1d and Supplementary Figure S1c). By contrast, the amounts of the Th2 signature cytokine, IL-13, were similar in WT and Trif $^{--}$mice. Only low amounts of interferon (IFN)$\gamma$ were seen, but $\mathrm{Trif}^{-1}$ mice had lower amounts of this cytokine in the lung than did WT mice when HDE-1, but not HDE-2, was used as an adjuvant. These observations suggest that at least in these models of asthma, TRIF is primarily required for adaptive immune pathways leading to production of IL-17 in the lung.

In addition to containing LPS and allergens (Supplementary Figure S1d), HDEs might also contain other microbial products other than LPS that signal through TRIF. To confirm that 
LPS-mediated allergic sensitization elicits neutrophilic asthmalike responses in a TRIF-dependent manner, we compared the abilities of WT and Trif $^{-1}$ mice to become sensitized to inhaled OVA when LPS is used as an adjuvant. Following instillation of LPS/OVA into the airways to sensitize mice, we elicited allergic airway inflammation by challenging the animals with aerosolized OVA (Figure 2a). As expected, WT mice developed airway neutrophilia and eosinophilia (Figure 2b). By comparison, Trif $^{-/}$mice had significantly reduced amounts of CXCL5 in the airway (data not shown), very few neutrophils, and a trend towards fewer eosinophils compared with WT mice (Figure 2b). Thus, in multiple models of allergic pulmonary inflammation, TRIF is critically required for the accumulation of airway neutrophils in response to allergen challenge. Lungs of OVA-challenged Trif ${ }^{-1}$ mice also had significantly less IL-17 and IL-13 than did lungs of WT mice (Figure 2c). Very little IFN- $\gamma$ was seen in either WT or Trif $^{-/}$mice. We also studied $M y d 88^{-/-}$mice and confirmed that they had marked reductions in both eosinophils and neutrophils (Figure 2b), as well as in all cytokines measured (Figure 2c), in agreement with previously reported results. ${ }^{29}$ Thus, Trif and Myd88 have non-redundant roles in this model of asthma.

We have previously shown using the LPS/OVA model of allergic asthma that conventional $\alpha \beta$ TCR $^{+}$Th17 cells are a
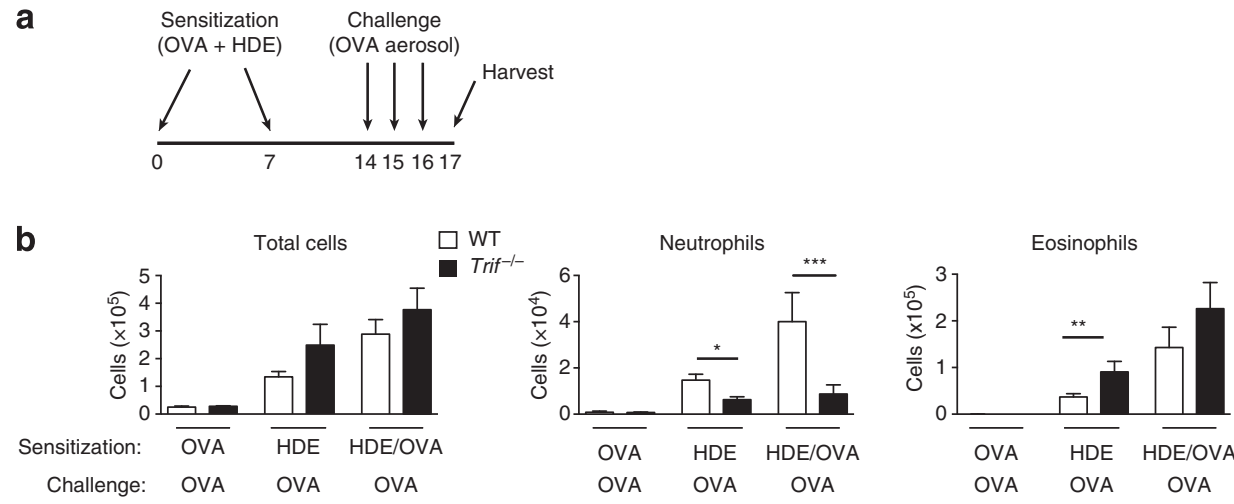

C
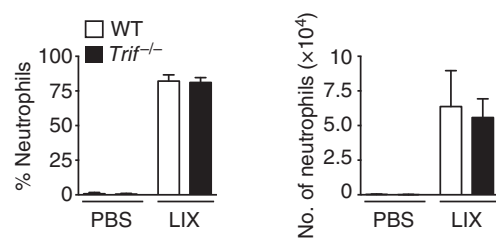

d
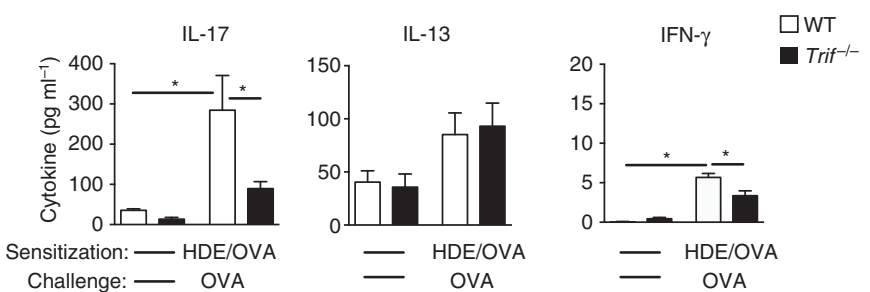

Figure 1 Trifis required for airway neutrophilia and interleukin (IL)-17 production in a house dust extract (HDE)-mediated model of asthma. (a) Timeline for allergic sensitization and challenge. (b) The mean number of leukocytes \pm s.e. in airways of ovalbumin (OVA)-challenged mice previously sensitized to OVA alone (OVA), $N=3$; HDE-1 alone (HDE) $N=11$, or HDE-1 plus OVA (HDE/OVA) $N=11$. Data shown are compiled from three independent experiments. (c) The mean number of neutrophils \pm s.e. in airways of WT and Trif ${ }^{-1}$ mice after CXCL5 inhalation. $N=3$. Data are from one experiment, representative of two. (d) Cytokines in supernatants of lungs excised with from HDE-1/OVA-sensitized and OVA-challenged mice. ${ }^{\star} P<0.05,{ }^{\star \star} P<0.01$, ${ }^{* * *} P<0.001$. Data shown are combined from two independent experiments. $N=5$ (untreated (dash) or 9 (HDE/OVA) per group.

Figure 2 TIR-domain-containing adapter-inducing interferon- $\beta$ (TRIF) is required for airway neutrophilia and T helper 17 (Th17) responses in a lipopolysaccharide (LPS)-mediated model of asthma. (a) Timeline of sensitization and challenge. (b) The mean number of leukocytes \pm s.e. in airways of

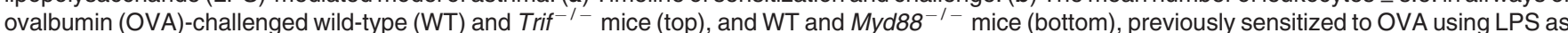
the adjuvant, or sensitized with OVA alone. $N=4$ (OVA alone) or 12 (LPS/OVA) group. Data shown are compiled from two experiments. (c) Cytokines in supernatants of lungs collected from LPS/OVA-sensitized and OVA-challenged mice, or untreated (Untr.) mice. (d,e) Intracellular staining of lung T cells for interleukin (IL)-17 (d) or IL-13 (e) 1 week after the second sensitization. Representative flow cytometry plots depicting the gating of cytokine-positive $\mathrm{CD}^{+}$T cells are shown, as well as histograms showing fold-increase in each cell type in LPS/OVA mice compared with OVA only mice. $N=6$ (OVA only) or 9 (LPS/OVA). (f) Inflammatory responses (left) and cytokine production (right) in lungs of WT and $/ I-1 r^{-1-}$ mice following sensitization with either OVA alone or LPS/OVA before challenge with aerosolized OVA. $N=8$ mice per group. Data are from a single experiment, representative of two. (g) Airway hyperresponsiveness (AHR) in WT and Trif $^{-1-}$ mice sensitized to OVA alone or OVA plus LPS, and challenged once with OVA. Data represent mean resistance $(\mathrm{R})$ values \pm s.e. from one experiment, representative of two. ${ }^{\star} P<0.05,{ }^{\star \star} P<0.01,{ }^{* \star \star} P<0.001 . N=8$ mice per group. 
a

Sensitization Challenge

(OVA +/- LPS) (OVA aerosol)

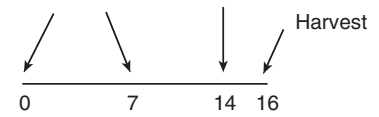

b

Total cells

Neutrophils

Eosinophils
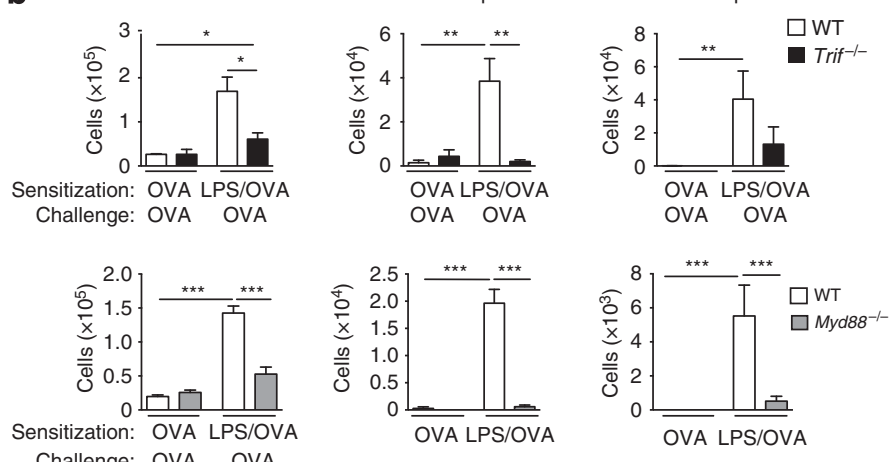

IL-17

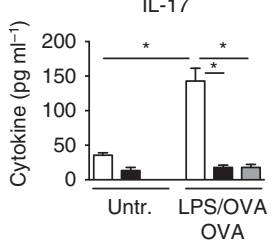

IL-13
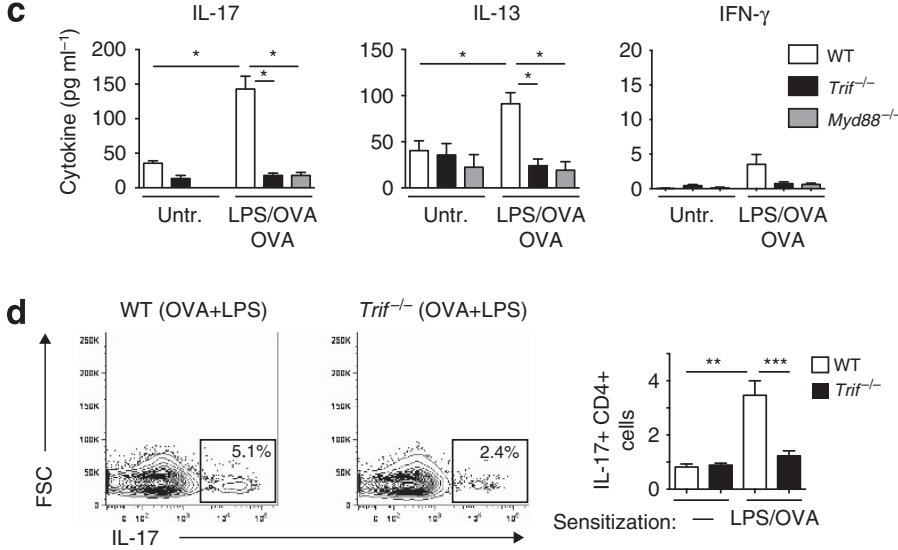

e
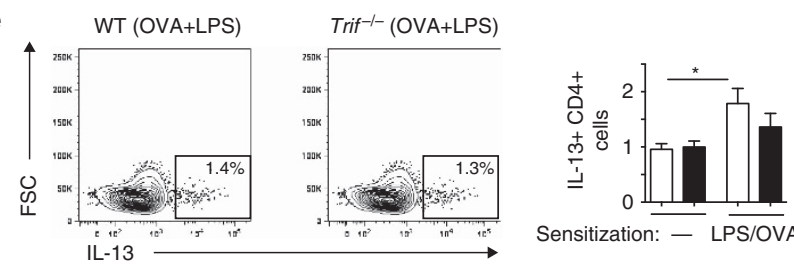

Sensitization: - LPS/OVA
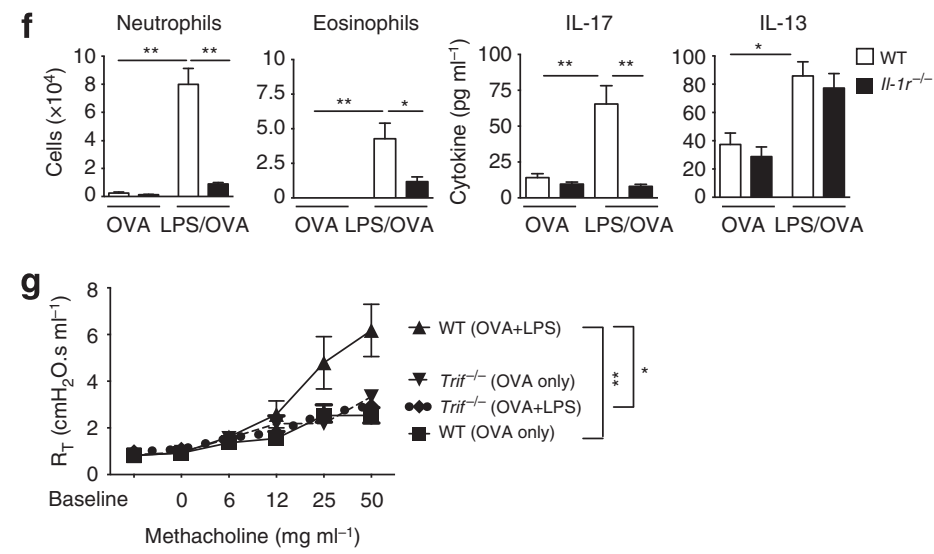
major source of IL-17, and that lesser amounts of this cytokine are produced by $\gamma \delta \mathrm{TCR}^{+} \mathrm{T}$ cells. ${ }^{22}$ To determine whether Trif $^{-1-}$ mice have fewer IL-17-containing Th17 cells than WT mice, we sensitized mice with LPS/OVA, prepared CD4 ${ }^{+} \mathrm{T}$ cells from their lungs, and performed intracellular cytokine staining for IL-17. We found that the percent and total number of Th17 cells in the lungs of rrif $^{-/}$mice were reduced compared with those of WT controls (Figure 2d), indicating that TRIF contributes to the induction of Th17 cells, or to their maintenance in the inflamed lung. By contrast, WT and Trif $^{\prime-}$ mice had similar numbers of IL-13-containing Th2 cells (Figure 2e). These data provide additional evidence that TRIF preferentially promotes Th17 cell accumulation and neutrophil recruitment to the airway in models of asthma.

IL- $1 \beta$, together with IL- 6 and transforming growth factor- $\beta$, induces the differentiation of Th17 cells from naive T cells. ${ }^{37}$ To determine the role of IL- $1 \beta$ in a model of neutophilic asthma, we studied mice lacking the receptor for this cytokine. After LPS/OVA sensitization and OVA challenge, $I l-1 r^{-/-}$mice had markedly fewer neutrophils than did WT mice (Figure 2f). Eosinophils were also reduced in $\mathrm{Il}-1 \mathrm{r}^{-/-}$mice, although less so than were neutrophils. IL-17 was reduced in lungs of $I l-1 r^{-/-}$ mice, but IL-13 was not, again suggesting a stronger effect of TRIF on Th17 responses than on Th2 responses.

Analyses of mucus production in the airway did not reveal differences between WT and Trif ${ }^{-1}$ mice (Supplementary Figure S2), in agreement with our previous observation that mice lacking IL-17RA develop strong mucus responses in this model. ${ }^{22}$ As previous studies have shown that pulmonary production of IL-17 contributes to AHR, a cardinal feature of allergic asthma, ${ }^{17,22,38}$ we used invasive measurements of airway resistance to investigate the requirement of TRIF for the development of AHR in the LPS/OVA model of asthma (Figure 2g). In untreated animals, no differences in methacholine responsiveness were seen between WT and Trif ${ }^{-1}$ mice. However, after LPS-mediated allergic sensitization and OVA challenge, WT mice developed much stronger responsiveness to methacholine, whereas Trif $f^{-1}$ mice did not. Thus, in addition to its requirement for IL-17-associated neutrophilic inflammation, TRIF is also required for AHR in this model of asthma.

\section{TRIF expression in hematopoietic cells is responsible for neutrophil recruitment to the lung during allergic inflammation}

Trif is expressed in a variety of cell types, including radiosensitive antigen-presenting cells such as macrophages and DCs, ${ }^{27,39}$ as well as radioresistant cells that include airway epithelial cells. ${ }^{40}$ To identify which of these cell compartments require Trif expression for Th17 development and neutrophil recruitment, we generated reciprocal bone marrow chimera mice using $\mathrm{WT}$ and Trif $^{-1}$ mice as donors and recipients, respectively. Following confirmation that donor cells had reconstituted at least $85 \%$ of the recipients' immune cells in the blood and lung, we sensitized the animals with LPS/OVA and challenged them with aerosolized OVA. As expected, neutrophils accumulated in the airways of WT mice receiving
WT marrow (WT $\rightarrow$ WT mice) after OVA challenge but were significantly reduced in $\mathrm{Trif}^{--} \rightarrow \mathrm{WT}$ mice. In addition, $\mathrm{WT} \rightarrow$ Trif $^{--}$mice had significantly more neutrophils in the airway than did Trif $^{-1} \rightarrow$ Trif $^{-1}$ mice (Figure 3a). Thus, Trif expression in hematopoietic cells is both necessary and sufficient for neutrophilic responses to allergen challenge. No significant differences in eosinophil number were seen among any of the groups, again suggesting that TRIF is not as critical for the accumulation of these cells in the lung as it is for neutrophil recruitment. Analysis of cytokines in lungs of the sensitized and challenged chimeric animals revealed that, similar to neutrophil accumulation in the airway, IL-17 production is also dependent on Trif expression in hematopoietic cells (Figure 3b). By contrast, the amounts of IL-5 and IFN- $\gamma$ in Trif $^{-1-} \rightarrow$ WT mice were similar to those of WT $\rightarrow$ WT mice. IL-4 production trended towards an increase in $\mathrm{Trif}^{-/} \rightarrow \mathrm{WT}$ mice, possibly because decreased Th17 development can be counterbalanced by increased IL-4 responses. Taken together, these data indicate that IL-17-associated neutrophilic inflammation in this model of asthma requires Trif expression in radiosensitive hematopoietic cells.

\section{Requirement of TRIF for lung DC migration and upregulation of CD40}

The requirement of Trif expression in hematopoietic cells for neutrophilic inflammation suggested that this adaptor protein might be important for the migration or function of lung DCs. These cells take up inhaled allergens, migrate to regional mediastinal lymph nodes, and present peptides to naive $\mathrm{T}$ cells in the context of upregulated costimulatory molecules and T-cell-activating cytokines. We tested the requirement of TRIF for each of these functions in lung DCs. We first used an established assay to test whether Trif expression is required for DC migration to lung-draining lymph nodes. In this assay, the fluorescent dye, $\mathrm{PKH}$, is instilled into the airways and taken up by DCs so that their migration to regional LNs can be tracked. Unlike carboxyfluorescein diacetate succinimidyl ester, $\mathrm{PKH}$ does not elicit inflammation and is not carried passively to LNs by the lymph. ${ }^{41}$ We found that regional LNs of mice that inhaled PKH alone contained a relatively low number of total CD11c ${ }^{+}$MHC class $\mathrm{II}^{\mathrm{hi}}$ DCs and $\mathrm{PKH}^{+}$migratory DCs, as would be expected during steady-state conditions (Figure 4a). By contrast, mice that had inhaled an adjuvant, either HDE or LPS, together with OVA and PKH had many more total DCs and migratory DCs in the LNs. Although the numbers of DCs were not statistically different between WT and Trif ${ }^{/-}$mice receiving $\mathrm{HDE} / \mathrm{PKH}$, Trif $^{--}$mice had a striking and statistically significant reduction in migratory DCs compared with WT mice after LPS/PKH treatment. The latter result suggests that the general decrease in inflammatory cytokines observed in lungs of $\mathrm{Trif}^{-1}$ mice treated with LPS/OVA (Figure 2c) is at least partly because of impaired migration of Trif $^{-1-}$ lung DCs during allergic sensitization.

The similar migration of WT and $\mathrm{Trif}^{-1}$ DCs to regional LNs after HDE instillation suggested that a difference between these two strains other than DC migration was likely responsible for 
a

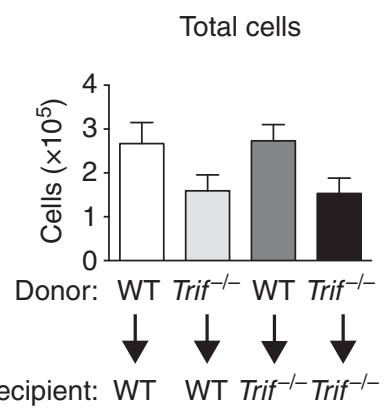

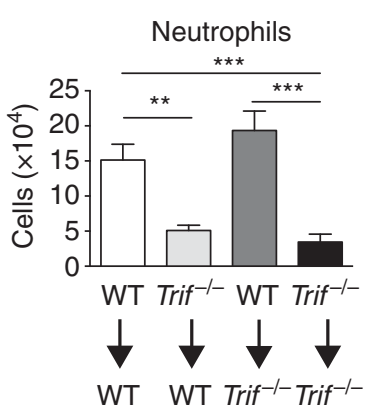

Eosinophils

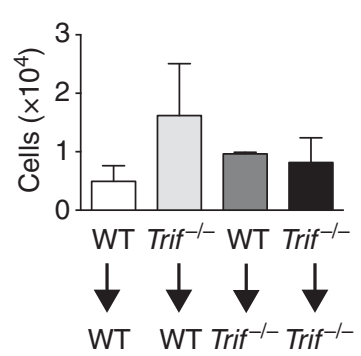

b

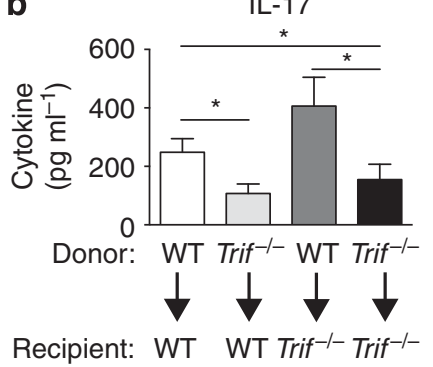

IFN- $\gamma$

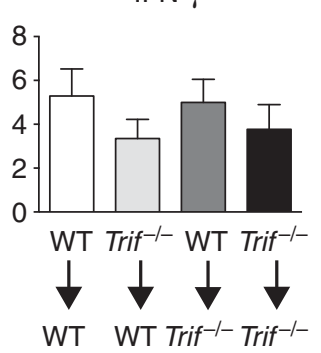

IL-4

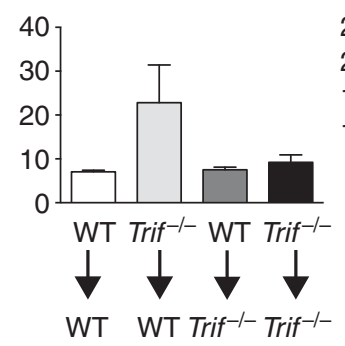

IL-5

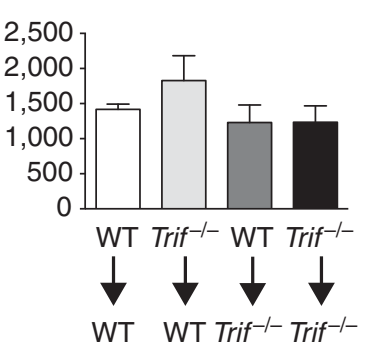

Figure 3 Trif expression on hematopoietic cells is required for neutrophil recruitment and interleukin (IL)-17 production in a lipopolysaccharide (LPS)mediated model of asthma. (a) The mean number of leukocytes \pm s.e. in airways of reciprocal bone marrow chimeric mice after LPS/ovalbumin (OVA) sensitization and OVA challenge. (b) Cytokines in supernatants of lungs cultured with OVA. ${ }^{\star} P<0.05,{ }^{\star \star} P<0.01,{ }^{\star \star *} P<0.001$. Data shown are combined from two independent experiments, $N=3-7$.

the striking decrease in IL-17 in HDE/OVA-treated mice (Figure 1d and Supplementary Figure S1c). To investigate potential mechanisms, we studied the display of costimulatory molecules on lung DCs. TRIF is required for LPS-induced upregulation of costimulatory molecules on thioglycollateelicited peritoneal macrophages, ${ }^{42}$ but not on splenic and bone marrow-derived DCs. ${ }^{43}$ This suggests that the requirement of TRIF in this regard might depend on the cell type being studied, prompting us to investigate whether Trif expression is required for LPS-induced upregulation of costimulatory molecules on lung DCs. To do this, we instilled LPS into the airways of WT and $\mathrm{Trif}^{-1-}$ mice and isolated non-autofluorescent, CD11c ${ }^{+}$ MHC class II ${ }^{\text {hi }}$ lung DCs on the following day (Figure $4 \mathbf{b}$ ). Analysis of costimulatory molecules using flow cytometry revealed that the mean fluorescent intensity of CD80 was similar in Trif $^{-1}$ and WT DCs (Figure 4c,d), but was decreased in $M y d 88^{-/-}$mice. CD86 was only slightly lower in $\mathrm{Trif}^{-1-} \mathrm{DCs}$, whereas the CD40 mean fluorescent intensity was significantly decreased in both $\mathrm{Trif}^{-1-}$ DCs and $\mathrm{Myd} 88^{-/-}$DCs compared with WT DCs.

\section{Stimulation of CD40 on lung DCs induces Trif-dependent production of Th17-skewing cytokines}

DCs instruct T-cell lineage development largely through their production of specific cytokines. Th17 development occurs in the presence of transforming growth factor- $\beta$ and IL- $6,{ }^{44,45}$ and IL-1 $\beta$ amplifies Th17 development. Although IL-23 is dispensable for the induction of Th17 cells, ${ }^{44}$ it enhances their longevity and pathogenicity. ${ }^{46}$ Activation of CD40 can trigger the production of Th17-promoting cytokines by some types of
DCs, ${ }^{47-51}$ but lung DCs have not been extensively studied in this regard. The reduced display of CD40 on lung DCs of Trif $^{\prime-}$ mice suggested that CD40-mediated production of Th17promoting cytokines by lung DCs might be impaired. To test this, we prepared total DCs from lungs of WT and Trif $^{-1}$ mice and evaluated their response to stimulation by anti-CD40 antibodies and LPS. This treatment increased the production of IL- $1 \beta$ and IL- 6 by WT DCs compared with untreated DCs, but failed to significantly increase the production of these same cytokines by Trif ${ }^{-1}$ DCs (Figure 4e). This suggests that the reduced display of CD40 on lung DCs from Trif $^{-1-}$ mice limits CD40-dependent production of Th17-promoting cytokines.

CD40L is rapidly upregulated on activated $\mathrm{T}$ cells and can trigger cytokine production by DCs through interactions with CD40. ${ }^{52}$ To determine whether Trif is required for T-cellmediated cytokine production by DCs, we instilled LPS/OVA into the lungs of WT and Trif ${ }^{--}$mice, prepared CD11 $\mathrm{c}^{+} \mathrm{I}-\mathrm{A}^{\mathrm{hi}}$ DCs and cultured them with naive, OVA-specific CD4 ${ }^{+} \mathrm{T}$ cells. Analysis of Th17 cell-promoting cytokines in the culture supernatants revealed that IL- 6 and IL- $1 \beta$ were significantly lower in cocultures that contained DCs from Trif ${ }^{/-}$mice than those containing WT DCs (Figure 4f).

\section{Lung DCs from Trif $^{-}$mice have decreased capacity to promote Th17 differentiation}

The reduced production of Th17-skewing cytokines by lung DCs from Trif $^{-1-}$ mice suggested that these DCs might have a reduced ability to promote Th17 cell differentiation compared with WT DCs. To test this, we instilled OVA/LPS into the airways of WT and $\mathrm{Trif}^{-1}$ mice to allow the protein to be taken 
a
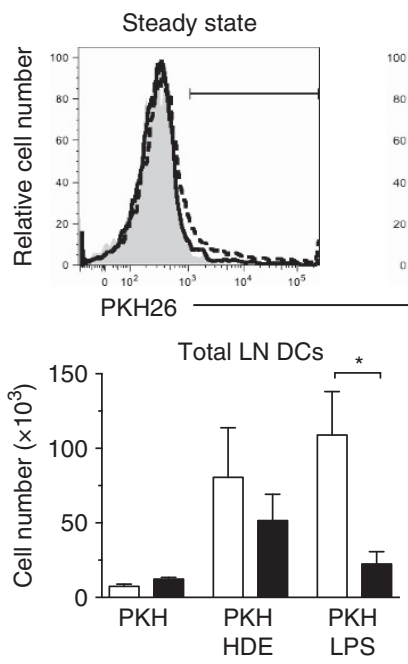

OVA/HDE
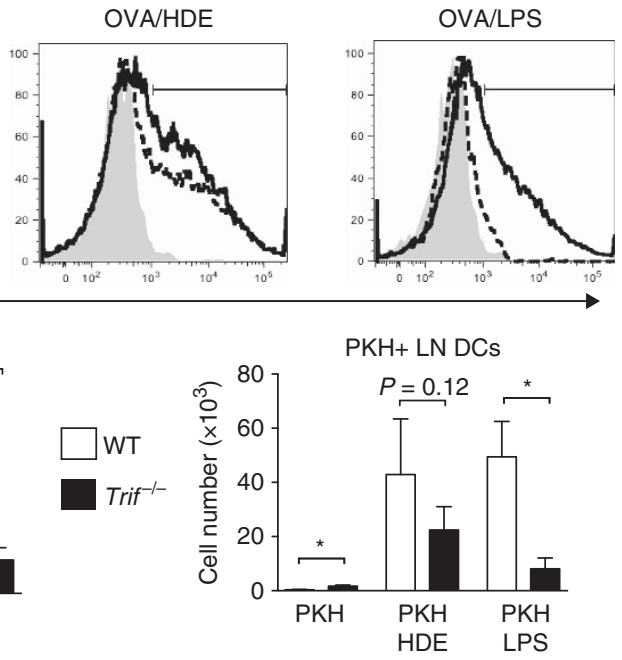

b

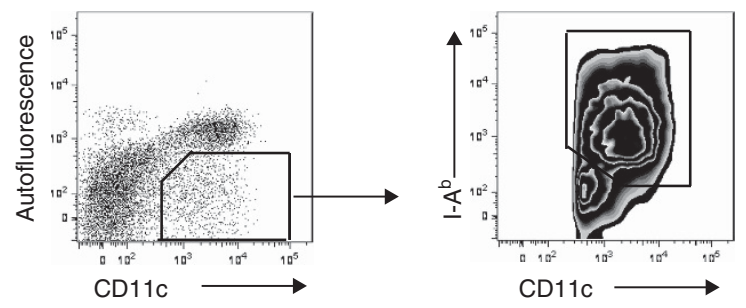

C
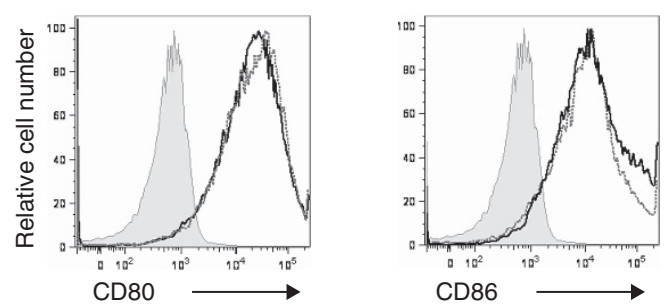$$
\text { ( }
$$
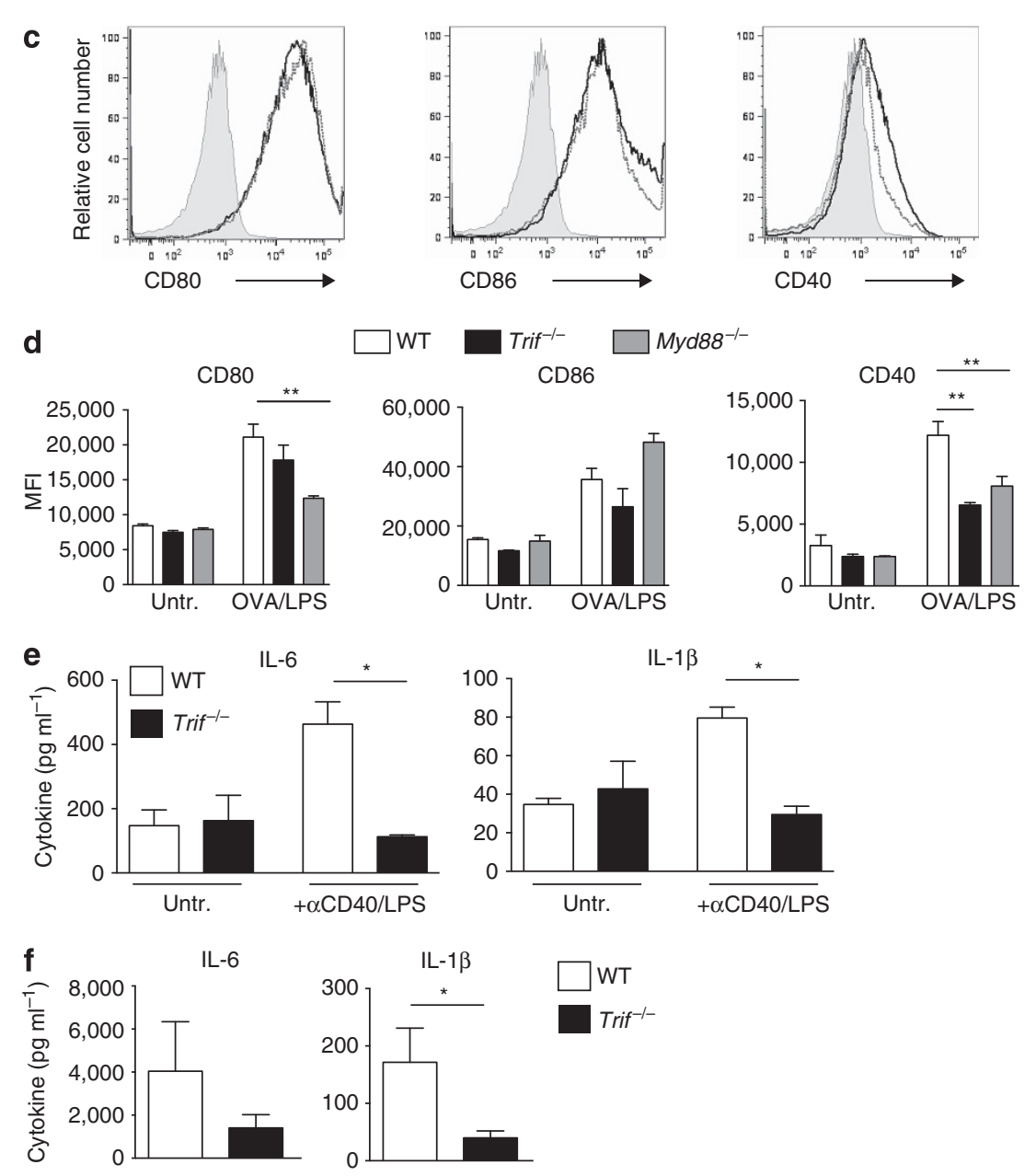
up by lung DCs in vivo, purified these cells, and cultured them again with OVA-specific, naive OT-II cells. A high DC:T cell ratio favors Th1 cell development, whereas lower ratios favor Th2 development. ${ }^{53,54}$ We therefore set up cultures with a high DC to $T$ cell ratio (1:1), as well as cultures with a low ratio (1:10). In agreement with previous studies, Th1 cytokines in the culture supernatants were highest in cultures with a high DC:T cell ratio (Figure 5a). Regardless of the culture conditions, however, DCs from $\mathrm{Trif}^{-1-}$ mice were severely compromised in their ability to induce Th1 and Th17 differentiations, whereas
Th2 cytokine production was unaffected by the absence of Trif in lung DCs. Intracellular staining confirmed that Th17 cell differentiation was impaired when Trif $^{-1}$ DCs were used (Figure 5b). Thus, Trif is required for lung DC-dependent induction of Th1 and Th17 differentiations, but dispensable for Th2 differentiation.

\section{DISCUSSION}

A growing body of evidence links Th17 cells and the cytokines they produce with steroid-resistant, neutrophilic asthma. ${ }^{11,13,14,55}$ a

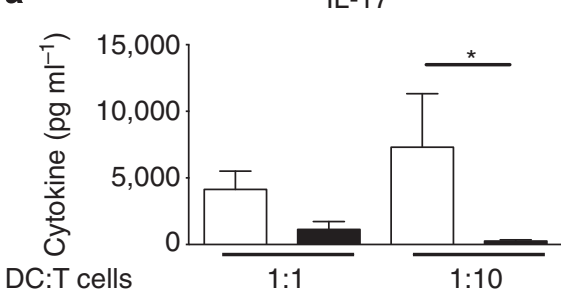

$\mathrm{IFN} \gamma$

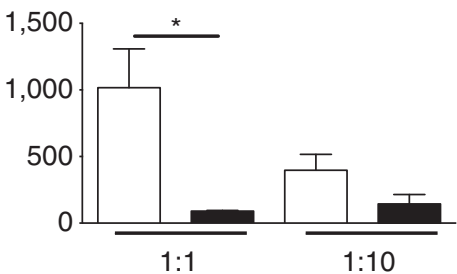

WT

Trif $^{-/-}$
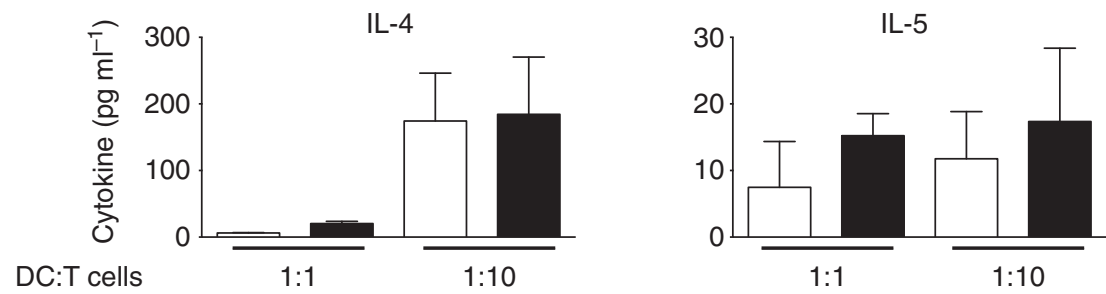

b

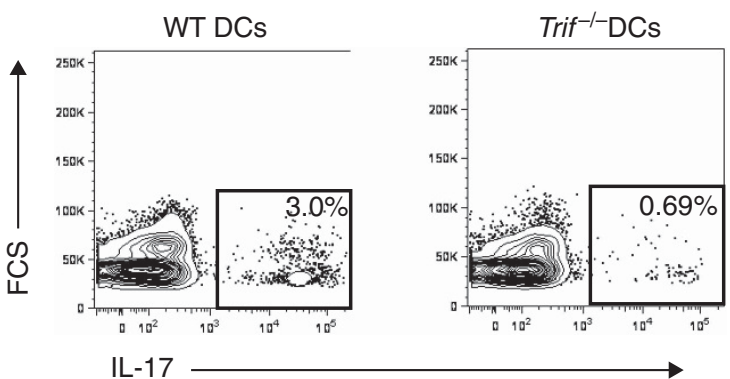

Figure 5 Trif-dependent induction of T helper 17 (Th17) differentiation by lung dendritic cells (DCs). Cytokine production (a) and intracellular staining (b) of OT-II CD4 ${ }^{+}$T cells, following their culture for 6 days with lung DCs of WT and Trif ${ }^{-1-}$ mice that had previously inhaled lipopolysaccharide (LPS)/ ovalbumin (OVA). ${ }^{*} P<0.05$. Data shown are combined from two independent experiments, $N=3-5$.

Figure 4 Requirement of TIR-domain-containing adapter-inducing interferon- $\beta$ (TRIF) for lung dendritic cell (DC) migration and upregulation of CD40. (a) Representative histograms showing PKH staining of CD11c ${ }^{+}$DCs from lymph nodes (LNs) of wild-type (WT) mice (solid lines) or Trif ${ }^{-1-}$ mice (dashed lines). Background fluorescence in mice not receiving PKH is indicated by the shaded region. Also shown are bar graphs indicating number of migratory, $\mathrm{PKH}^{+} \mathrm{DCs}$ in LNs of WT and Trif ${ }^{-1-}$ mice. Data are from one experiment. (b) Gating strategy for lung DCs, based on their display of CD11C and major histocompatibility complex (MHC) class II and lack of autofluorescence. (c,d) Costimulatory molecule display on lung DCs following lipopolysaccharide (LPS)/ovalbumin (OVA) inhalation. (c) Representative flow cytometry plots showing isotype control antibody staining as a shaded region, specific staining of WT DCs as a black line, and specific staining of Trif $^{-1}-$ DCs as a grey line. (d) The mean fluorescent intensity \pm s.e. of specific staining for the indicated costimulatory molecule on lung DCs from untreated (Untr.) or OVA/LPS-treated WT, Trif ${ }^{-1-}$ and $M_{y d 88^{-1-}}$ mice. Data are from a single experiment, representative of two. $N=3$ (untreated) to 6 (LPS-treated) mice per group. (e) The mean amounts of Thelper 17 (Th17)-promoting cytokines in supernatants of untreated lung DCs (Untr), or DCs cultured with LPS and antibodies against CD40. (f) Th17-promoting cytokines in supernatants of lung DCs prepared from WT and Trif ${ }^{-1}$ mice and cultured with OVA and OVA-specific T cells. ${ }^{\star} P<0.05,{ }^{* *} P<0.01$. Data shown are combined from two independent experiments, $N=3-6$. 
Individuals with this form of asthma represent an unmet clinical need, and there is a critical need for novel therapeutic approaches. Although LPS, including inhaled LPS, has been related to the development of Th17 responses, ${ }^{20,22,56}$ the basis for its potency in this regard has been unclear. A better understanding of LPS-induced Th17 responses might lead to novel interventional strategies. Data from the present study identify a Trif-dependent pathway leading from microbial product recognition to the development of Th17 cells, which are in turn required for neutrophilic inflammation of the airway and AHR. We found that MyD88 and TRIF are each required for the development of Th17 responses to inhaled allergens, suggesting that these two adaptor molecules have one or more unique or synergistic actions that affect the Th17 developmental pathway. This interpretation is consistent with a recent analysis of MyD88- and TRIF-dependent proteins secreted from LPS-activated macrophages. ${ }^{28}$ That report revealed that, although most proteins require only one of the two pathways for production and/or secretion, other proteins require both adaptors, while still others have a specific requirement for either TRIF or MyD88.

We found that Trif was required for the upregulation of CD40 on lung DCs in vivo, and that stimulation of this molecule with either anti-CD40 antibodies or with $\mathrm{CD}^{+} \mathrm{T}$ cells triggered production of the Th17-promoting cytokines, IL-1 $\beta$ and IL- 6 ex vivo. In turn, production of IL- $1 \beta$ was required for airway neutrophilia because it failed to develop in ${\mathrm{Il}-1 r^{-/-}}$mice. This result suggests a role of the inflammasome and activation of caspase- 1 , which cleaves the precursor form of IL- $1 \beta$ into its active form. Previous reports have shown that LPS can induce If $n$ - $\beta$ expression by a TRIF- and interferon regulatory factor (IRF)-3-dependent mechanism, ${ }^{57}$ and we confirmed that Ifn- $\beta$ is increased by OVA-LPS inhalation in a TRIF-dependent manner (unpublished observations). Although Irf-3 mRNA was not increased (unpublished observations), IRF-3 function can be governed by post-translational modifications, including phosphorylation, which directs this protein to the nucleus and might be important in Trif-dependent Th17 induction and neutrophilia. It has also been established that upregulation of costimulatory molecules is at least partly dependent on type I interferons. ${ }^{42}$ A plausible role for TRIF in the induction of Th17 responses by LPS might therefore involve sequential activation of IRF-3, induction of type I interferons, upregulation of CD40, and finally release of IL- 6 and IL- $1 \beta$ following engagement of CD40 on DCs by CD40L on T cells. We did not detect IL-23 in supernatants of DC cultures, but IRF-3 is reported to bind the human IL-23p19 promoter in monocytes in response to TLR3 signaling. ${ }^{51}$ It is therefore possible that TRIF promotes Th17 survival and expansion in vivo by increasing levels of IL-23 in lung DCs or accessory cells.

In addition to promoting IL- $1 \beta$ and IL- 6 release by augmenting $\mathrm{CD} 40 \mathrm{~L} / \mathrm{CD} 40$ interactions, TRIF might also contribute to Th17 responses to inhaled allergens by increasing recruitment to the lung of DCs with the potential to drive Th17 cell differentiation. Although similar numbers of $\mathrm{CD} 11 \mathrm{~b}^{+} \mathrm{DCs}$ were seen in the lungs of untreated WT and Trif ${ }^{-1-}$ mice, the latter strain had fewer CD11b ${ }^{+}$DCs after LPS inhalation (unpublished observation). Monocyte-derived, Ly6G $^{+}$ $\mathrm{CD} 14^{\mathrm{hi}} \mathrm{CD} 11 \mathrm{~b}^{+}$inflammatory DCs are rapidly recruited to the lung during acute pulmonary inflammation, including LPSmediated inflammation, ${ }^{41,58}$ and human inflammatory DCs are reported to induce Th17 differentiation..$^{59}$ The monocyteattracting chemokine, CCL12, might be important for this because TRIF is required for secretion of CCL12 by LPSactivated macrophages. ${ }^{28}$ Pre-DC-derived $\mathrm{CD} 14^{\mathrm{lo}} \mathrm{CD} 11 \mathrm{~b}^{+}$ conventional DCs are also increased in the lungs of mice following LPS inhalation. ${ }^{41}$ These DCs are probably similar to $\mathrm{CD} 11 \mathrm{~b}^{+} \mathrm{CD} 64^{-} \mathrm{CD} 24^{+}$conventional DCs that were recently reported to control Th17 responses to Aspergillus fumigatus. ${ }^{60}$ Additional studies will be needed to determine whether the decreased ability of total lung DCs from Trif ${ }^{-1-}$ mice to promote Th17 differentiation in vitro is due in part to their reduced frequency of $\mathrm{CD} 11 \mathrm{~b}^{+}$DCs in those cultures.

We consistently observed striking reductions in the accumulation of IL-17 and neutrophils in lungs of allergen-challenged Trif $^{-1-}$ mice compared with WT mice, regardless of the specific model of asthma employed. In addition, lung DCs from Trif $^{-1-}$ mice were almost totally deficient in their ability to promote Th17 differentiation. Together, these data strongly support a role of TRIF in the induction of Th17 responses to inhaled allergens. Lung DCs from Trif $^{-1-}$ mice were also compromised in their ability to support Th1 differentiation ex vivo, and WT and Trif ${ }^{-1-}$ mice produced similar amounts of IFN- $\gamma$ in the lung when HDE-1 was used for sensitization, but not when HDE-2 was used. We have not yet investigated whether most of the IFN- $\gamma$ produced in the lung are from Th1 cells, or other cells, such as natural killer or natural killer T cells. ${ }^{61}$ With regard to the induction of Th2 responses, evidence from the present study indicates that Trif is generally dispensable. When two different HDEs were used individually to promote allergic responses to OVA, WT and Trif $^{-1-}$ mice produced similar amounts of IL- 4 and IL- 5 and had similar numbers of eosinophils in the airway. In addition, lung DCs from Trif $^{-1-}$ and WT mice were almost identical in their abilities to drive Th2 differentiation ex vivo. These data suggest that Trif expression in DCs is dispensable for Th2 differentiation. However, eosinophil accumulation in the airway and pulmonary production of Th2 cytokines were decreased in Trif $^{-1-}$ mice when LPS was the sole adjuvant used to prime allergic sensitization to OVA. The latter observation was likely due-at least in part-to markedly reduced trafficking of pulmonary DCs to regional LNs of Trif $^{-1-}$ mice following LPS/OVA instillation. A smaller and statistically insignificant reduction in DC migration was observed in Trif $^{-1-}$ mice after HDE/OVA instillation. Together, these data suggest that when LPS is the sole adjuvant in the airway, TRIF signaling is critical to efficient lung DC migration to regional LNs, but that unidentified products in HDEs can promote DC migration in a TRIF-independent manner.

Our finding that TRIF is critical for the development of Th17 responses to inhaled allergens is in agreement with a recent 
report showing that, although Trif $^{-1-}$ mice have exacerbated disease in an allergen-induced model of eczema, they have reduced amounts of IL-17A and IL-17F. ${ }^{62}$ Conversely, Trif $^{-1-}$ mice have increased IL-17 production and more severe disease in a model of experimental autoimmune encephalomyelitis, ${ }^{63}$ and splenic DCs from Trif ${ }^{-1-}$ mice have increased production of cytokines and display of costimulatory molecules after stimulation with various TLR agonists. ${ }^{64}$ Thus, the impact of TRIF on stimulatory and regulatory pathways might depend on the target organ and animal model used. Additional studies will be required to test this possibility. It will be informative to learn whether small molecule-mediated inhibition of the TRIFdependent signaling pathway can reduce Th17 responses to inhaled allergens. Several TRIF-specific inhibitors have already been identified; ${ }^{65-67}$ however, the ultimate usefulness of such molecules for treating neutrophilic asthma will depend on whether they are able to attenuate allergen-specific Th17 cell development without inhibiting immune responses required for host defense in the lung or other organs.

\section{METHODS}

Animals. Trif-deficient $\left(\right.$ Trif $\left.^{--}\right)$mice were obtained from Shizuo Akira (Osaka University), backcrossed for 10 generations to C57BL/6 and maintained as homozygotes. The following strains were purchased from Jackson Laboratories (Bar Harbor, ME): C57BL/6J,

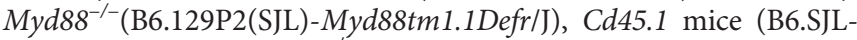
Ptprca Pepcb/BoyJ) Il1r1 ${ }^{-/-}$(B6.129S7-Il1r1tm1Imx/J), and OT-II mice (B6.Cg-Tg(TcraTcrb)425Cbn/J). C57BL/6J used for WT controls were either purchased from Jackson Laboratories and housed for 3 weeks at the National Institute for Environmental Health Sciences (NIEHS), or bred alongside Trif ${ }^{1-}$ mice. Experimental mice were ageand sex-matched, and used at 6-12 weeks of age in accordance with the guidelines provided by the Institutional Animal Care and Use Committee at the NIEHS.

House dust extracts. The two HDEs used in this study have been described previously. ${ }^{68}$ Each HDE contains dust mite allergens but with different endotoxin activities. One extract (HDE-1) has an endotoxin activity of 2,000 endotoxin units (EU) per ml (limulus amebocyte lysate (LAL) assay, Lonza, Karlsruhe, Germany), approximately equal to $10^{-2} \mu \mathrm{g}$ LPS per $20 \mu \mathrm{l} \mathrm{HDE}$. The other extract, HDE-2, had an endotoxin activity approximately equal to $10^{-1} \mu \mathrm{g}$ LPS per $20 \mu \mathrm{l}$ HDE. Allergens were measured using a multiplex array for indoor allergens (Indoor Biotechnologies, Charlottesville, VA), according to the manufacturers' instructions.

LPS- and HDE-mediated allergic airway disease. On days 0 and 7 , mice were anesthetized with inhaled isoflurane and sensitized by oropharyngeal aspirations $^{22}$ of LPS-depleted OVA (catalog no. 321000; BioVendor, Candler, NC) alone or together with either $100 \mathrm{ng}$ of Escherichia coli LPS (catalog no. L2630; Sigma Chemical, St Louis, MO), or together with $20 \mu \mathrm{l}$ of an HDE. Asthma-like responses were subsequently elicited in sensitized mice by exposing them to nebulized (Ultr-Neb99; DeVilbiss Healthcare, Somerset, PA) 1\% OVA (catalog no. A5503; Sigma Chemical Company) in saline. Mice sensitized with HDE/OVA were challenged with OVA for $30 \mathrm{~min}$ on days 14-16, and subjected to BAL $24 \mathrm{~h}$ after the final challenge. Mice sensitized with LPS/OVA were challenged for $1 \mathrm{~h}$ on day 14 and harvested $48 \mathrm{~h}$ post challenge. Following BAL, the large lobe of the lung was excised, placed into sterile RPMI 1640 containing 10\% FBS and OVA (Sigma Chemical) at a concentration of $10 \mu \mathrm{g} \mathrm{ml}^{-1}$ and cultured for $24 \mathrm{~h}$ at $37^{\circ} \mathrm{C}$ with $5 \% \mathrm{CO}_{2}$. Cell culture fluids were centrifuged, and the supernatants stored at $-80{ }^{\circ} \mathrm{C}$ until analysis. Cytokines were measured using a multiplexed fluorescent bead-based immunoassay (Bio-Plex; Bio-Rad laboratories, Hercules, CA) according to the manufacturer's instructions.

Cellular analysis. Differential analysis of cells in BAL fluid was performed as described previously. ${ }^{69}$ For analyses of intracellular cytokines, cells were stimulated with $50 \mathrm{ng} \mathrm{ml}^{-1}$ phorbol myristate acetate and $500 \mathrm{ng} \mathrm{ml}^{-1}$ ionomycin (Sigma) for $4 \mathrm{~h}$ before staining, and incubated with GolgiStop during the last $3 \mathrm{~h}$ of stimulation. Cells were fixed and permeabilized using Cytofix/Cytoperm (BD Biosciences, San Jose, CA), then stained with the antibodies against IL-13 (eBio13A, eBiosciences, San Diego, CA), and IL-17 (clone eBio17B7, eBioscience, or clone TC11-18H10, BD Biosciences). Stained cells were acquired on a fluorescence-activated cell sorting LSRII flow cytometer using the Diva software (BD Biosciences), and the data were analyzed with the FlowJo software (Tree Star, Ashland, OR).

Histology. Lungs were fixed in $10 \%$ formalin and embedded in paraffin. Left lobe longitudinal sections 5- to $7-\mu \mathrm{m}$ thick were stained with Alcian Blue together with Periodic acid-Schiff, visualized with an Olympus BX51 microscope and photographed using an Olympus DP70 digital camera.

AHR analysis. For AHR evaluation, mice were anesthetized by intraperitoneal injection with urethane $\left(1.5 \mathrm{~g} \mathrm{~kg}^{-1}\right)$, paralyzed by intraperitoneal injection with pancuronium bromide $\left(0.8 \mathrm{mg} \mathrm{kg}^{-1}\right)$, and intubated. AHR was analyzed using the FlexiVent mechanical ventilator system (Scireq; Montreal, Quebec, Canada) as previously described. $^{22}$ We measured total respiratory resistance and report peak values.

Generation of reciprocal bone marrow chimeric mice. WT (CD45.1) and Trif $^{-1}$ (CD45.2) mice were used so that donor and host cells could be distinguished from one another. Recipient mice were lethally irradiated using a Cesium irradiator (9.25 Gy over $12 \mathrm{~min}$ ), and were injected intraventrally with $10^{7}$ bone marrow cells. At 13 weeks post irradiation, antibodies against CD45.1 (A20) and CD45.2 (104) were used to assess donor engraftment in T cells (anti-CD3), B cells (antiCD19; 6D5), myeloid cells, anti-CD11b (M1/70), and DCs and alveoloar macrophages (anti-CD11c; N418 and HL3), BD Biosciences, BioLegend, and eBioscience. At least $85 \%$ reconstitution was observed for each cell population tested.

Analysis of lung DCs. Lung DC migration assays using $\mathrm{PKH}$ as a fluorescent tag were carried out as described previously. ${ }^{4 \mathrm{P}}$ To analyze costimulatory molecules on lung DCs, mouse airways were instilled with OVA plus LPS, and the animals were killed $16 \mathrm{~h}$ post treatment with an overdose of pentobarbital sodium. Following perfusion of the pulmonary vasculature with phosphate-buffered saline, lungs were excised and placed in ice-cold phosphate-buffered saline containing $0.5 \%$ bovine serum albumin. DCs from minced and digested lung tissue were isolated as previously described ${ }^{70}$ and stained with antibodies against I-A ${ }^{b}$ and CD11c. For analysis of costimulatory marker expression, DCs were evaluated with flow cytometry using antibodies against the following molecules: I-A ${ }^{\mathrm{b}}$ (AFb.120) CD103 (M290), CD11c (N418), CD11b (M1/70), CD40 (Ic10), CD80 (1610A1), and CD86 (GL1), BD Biosciences, BioLegend, and eBioscience.

Isolation and culture of lung DCs. Mouse airways were instilled with phosphate-buffered saline or LPS-free OVA plus $100 \mathrm{ng}$ LPS. At $16 \mathrm{~h}$ post treatment, non-autofluorescent, $C D 11 c^{+} \mathrm{I}-\mathrm{A}^{\mathrm{b}}$ hi $\mathrm{DCs}$ were isolated as described above and sorted on a FACS Aria (BD Biosciences). For CD40 stimulation, lung DCs were plated at a concentration of 125,000 cells per $200 \mu \mathrm{l}$ in a 96-well plate with or without LPS (100 $\mathrm{ng} \mathrm{ml}^{-1}$ ) and $10 \mu \mathrm{g} \mathrm{ml}^{-1}$ plate bound anti-CD40 (FGK45.5; eBioscience). Cells were incubated overnight at $37^{\circ} \mathrm{C}$ and supernatants were collected and analyzed for cytokines as described above. For DC/ $\mathrm{T}$ cell cocultures, DCs were cultured together with naive $\mathrm{T}$ cells prepared from the spleen and lymph nodes from OT-II donor mice as 
described previously. ${ }^{70}$ Supernatants were collected after 6 days of culture and analyzed for production of DC and T-cell-specific cytokines as described above.

Statistical analysis. Statistical analyses were performed using the Prism software (GraphPad Software). Data are expressed as mean \pm s.e. When two group comparisons were made, the Student's $t$-test was used. A repeated measures analysis of variance with the Bonferroni post hoc test was used to determine differences in AHR. Before any statistical analyses, outliers were excluded using the Grubb's test. Each figure legend specifies the number of samples for the experiments and the level of significance of each indicated comparison. $P$-values of less than 0.05 were considered statistically significant.

SUPPLEMENTARY MATERIAL is linked to the online version of the paper at http://www.nature.com/mi

\section{ACKNOWLEDGMENTS}

We thank Maria Sifre and Carl Bortner for help with flow cytometry, Ligon Perrow for support with animal experiments, and Michael Fessler (NIEHS) for critical reading of the manuscript. This work was supported by the Intramural Research Program of the National Institutes of Health $(\mathrm{NIH})$ and the NIEHS.

\section{DISCLOSURE}

The authors declared no conflict of interest.

c 2015 Society for Mucosal Immunology

\section{REFERENCES}

1. Martinez, F.D. \& Vercelli, D. Asthma. Lancet 382, 1360-1372 (2013).

2. Wenzel, S.E. Eosinophils in asthma-closing the loop or opening the door? N. Engl. J. Med. 360, 1026-1028 (2009).

3. Larche, M., Robinson, D.S. \& Kay, A.B. The role of T lymphocytes in the pathogenesis of asthma. J. Allergy Clin. Immunol. 111, 450-463 (2003).

4. Green, R.H., Brightling, C.E., Woltmann, G., Parker, D., Wardlaw, A.J. \& Pavord, I.D. Analysis of induced sputum in adults with asthma: identification of subgroup with isolated sputum neutrophilia and poor response to inhaled corticosteroids. Thorax 57, 875-879 (2002).

5. Haldar, P. \& Pavord, I.D. Noneosinophilic asthma: a distinct clinical and pathologic phenotype. J. Allergy Clin. Immunol. 119, 1043-1052 (2007).

6. Pavord, I.D., Brightling, C.E., Woltmann, G. \& Wardlaw, A.J. Non-eosinophilic corticosteroid unresponsive asthma. Lancet 353, 2213-2214 (1999).

7. Fahy, J.V., Kim, K.W., Liu, J. \& Boushey, H.A. Prominent neutrophilic inflammation in sputum from subjects with asthma exacerbation. J. Allergy Clin. Immunol. 95, 843-852 (1995).

8. Wenzel, S.E. et al. Evidence that severe asthma can be divided pathologically into two inflammatory subtypes with distinct physiologic and clinical characteristics. Am. J. Respir. Crit. Care Med. 160, 1001-1008 (1999).

9. Woodruff, P.G. et al. Relationship between airway inflammation, hyperresponsiveness, and obstruction in asthma. J. Allergy Clin. Immunol. 108 753-758 (2001).

10. Hastie, A.T. et al. Analyses of asthma severity phenotypes and inflammatory proteins in subjects stratified by sputum granulocytes. J. Allergy Clin. Immunol. 125, 1028-1036 e1013 (2010).

11. Barczyk, A., Pierzchala, W. \& Sozanska, E. Interleukin-17 in sputum correlates with airway hyperresponsiveness to methacholine. Respir. Med. 97, 726-733 (2003).

12. Bullens, D.M. et al. IL-17 mRNA in sputum of asthmatic patients: linking T cell driven inflammation and granulocytic influx? Respir. Res. 7, 135 (2006).

13. Kaminska, M. et al. Airway remodeling in subjects with severe asthma with or without chronic persistent airflow obstruction. J. Allergy Clin. Immunol. 124, 45-51 (2009).
14. Molet, S. et al. IL-17 is increased in asthmatic airways and induces human bronchial fibroblasts to produce cytokines. J. Allergy Clin. Immunol. 108, 430-438 (2001).

15. Liang, S.C. et al. An IL-17F/A heterodimer protein is produced by mouse Th17 cells and induces airway neutrophil recruitment. J. Immunol. 179, 7791-7799 (2007).

16. Kudo, M. et al. IL-17A produced by alphabeta T cells drives airway hyperresponsiveness in mice and enhances mouse and human airway smooth muscle contraction. Nat. Med. 18, 547-554 (2012).

17. McKinley, L. et al. TH17 cells mediate steroid-resistant airway inflammation and airway hyperresponsiveness in mice. J. Immunol. 181, 4089-4097 (2008).

18. Fedele, G. et al. Lipopolysaccharides from Bordetella pertussis and Bordetella parapertussis differently modulate human dendritic cell functions resulting in divergent prevalence of Th17-polarized responses. J. Immunol. 181, 208-216 (2008)

19. McAleer, J.P. et al. Potent intestinal Th17 priming through periphera lipopolysaccharide-based immunization. J. Leukoc. Biol. 88, 21-31 (2010).

20. Shi, G. et al. Differential involvement of Th1 and Th17 in pathogenic autoimmune processes triggered by different TLR ligands. J. Immunol. 191, 415-423 (2013).

21. Wang, L. et al. High dose lipopolysaccharide triggers polarization of mouse thymic Th17 cells in vitro in the presence of mature dendritic cells. Cell Immunol. 274, 98-108 (2012).

22. Wilson, R.H., Whitehead, G.S., Nakano, H., Free, M.E., Kolls, J.K. \& Cook, D.N. Allergic sensitization through the airway primes Th17dependent neutrophilia and airway hyperresponsiveness. Am. J. Respir. Crit. Care Med. 180, 720-730 (2009).

23. Wilson, R.H. et al. The Toll-like receptor 5 ligand flagellin promotes asthma by priming allergic responses to indoor allergens. Nat. Med. 18 , 1705-1710 (2012)

24. Ross, M.A. et al. Association of asthma symptoms and severity with indoor bioaerosols. Allergy 55, 705-711 (2000).

25. Thorne, P.S., Kulhankova, K., Yin, M., Cohn, R., Arbes, S.J. Jr. \& Zeldin, D.C. Endotoxin exposure is a risk factor for asthma: the national survey of endotoxin in United States housing. Am. J. Respir. Crit. Care Med. 172 1371-1377 (2005)

26. Braun-Fahrlander, C. et al. Environmental exposure to endotoxin and its relation to asthma in school-age children. N. Engl. J. Med. 347, 869-877 (2002).

27. Yamamoto, M. et al. Role of adaptor TRIF in the MyD88-independent tolllike receptor signaling pathway. Science 301, 640-643 (2003).

28. Meissner, F., Scheltema, R.A., Mollenkopf, H.J. \& Mann, M. Direct proteomic quantification of the secretome of activated immune cells. Science 340, 475-478 (2013).

29. Piggott, D.A. et al. MyD88-dependent induction of allergic Th2 responses to intranasal antigen. J. Clin. Invest. 115, 459-467 (2005).

30. Boasen, J., Chisholm, D., Lebet, L., Akira, S. \& Horner, A.A. House dust extracts elicit Toll-like receptor-dependent dendritic cell responses. J. Allergy Clin. Immunol. 116, 185-191 (2005).

31. McKinley, L., Kim, J., Bolgos, G.L., Siddiqui, J. \& Remick, D.G. Reproducibility of a novel model of murine asthma-like pulmonary inflammation. Clin. Exp. Immunol. 136, 224-231 (2004).

32. Lam, D., Ng, N., Lee, S., Batzer, G. \& Horner, A.A. Airway house dust extract exposures modify allergen-induced airway hypersensitivity responses by TLR4-dependent and independent pathways. J. Immunol. 181, 2925-2932 (2008).

33. Ng, N., Lam, D., Paulus, P., Batzer, G. \& Horner, A.A. House dust extracts have both $\mathrm{TH} 2$ adjuvant and tolerogenic activities. J. Allergy Clin. Immunol. 117, 1074-1081 (2006).

34. Wingender, G. et al. Invariant NKT cells are required for airway inflammation induced by environmental antigens. J. Exp. Med. 208, 1151-1162 (2011).

35. Whitehead, G.S., Thomas, S.Y. \& Cook, D.N. Modulation of distinct asthmatic phenotypes in mice by dose-dependent inhalation of microbial products. Environ. Health Perspect. 122, 34-42 (2014).

36. Eisenbarth, S.C., Piggott, D.A., Huleatt, J.W., Visintin, I., Herrick, C.A. \& Bottomly, K. Lipopolysaccharide-enhanced, toll-like receptor 4-dependent T helper cell type 2 responses to inhaled antigen. J. Exp. Med. 196, 1645-1651 (2002). 
37. Chung, Y. et al. Critical regulation of early Th17 cell differentiation by interleukin-1 signaling. Immunity 30, 576-587 (2009).

38. Lajoie, S. et al. Complement-mediated regulation of the IL-17A axis is a central genetic determinant of the severity of experimental allergic asthma. Nat. Immunol. 11, 928-935 (2010).

39. Weighardt, $\mathrm{H}$. et al. Identification of a TLR4- and TRIF-dependent activation program of dendritic cells. Eur. J. Immunol. 34, 558-564 (2004).

40. Zhu, L., Lee, P.K., Lee, W.M., Zhao, Y., Yu, D. \& Chen, Y. Rhinovirusinduced major airway mucin production involves a novel TLR3-EGFRdependent pathway. Am. J. Respir. Cell Mol. Biol. 40, 610-619 (2009).

41. Nakano, $\mathrm{H}$. et al. Migratory properties of pulmonary dendritic cells are determined by their developmental lineage. Mucosal Immunol. 6, 678-691 (2013).

42. Hoebe, K. et al. Upregulation of costimulatory molecules induced by lipopolysaccharide and double-stranded RNA occurs by Trif-dependent and Trif-independent pathways. Nat. Immunol. 4, 1223-1229 (2003).

43. Shen, H., Tesar, B.M., Walker, W.E. \& Goldstein, D.R. Dual signaling of MyD88 and TRIF is critical for maximal TLR4-induced dendritic cell maturation. J. Immunol. 181, 1849-1858 (2008).

44. Veldhoen, M., Hocking, R.J., Atkins, C.J., Locksley, R.M. \& Stockinger, B. TGFbeta in the context of an inflammatory cytokine milieu supports de novo differentiation of IL-17-producing T cells. Immunity 24, 179-189 (2006).

45. Bettelli, E. et al. Reciprocal developmental pathways for the generation of pathogenic effector TH17 and regulatory T cells. Nature 441, 235-238 (2006).

46. McGeachy, M.J. et al. The interleukin 23 receptor is essential for the terminal differentiation of interleukin 17-producing effector T helper cells in vivo. Nat. Immunol. 10, 314-324 (2009).

47. lezzi, G., Sonderegger, I., Ampenberger, F., Schmitz, N., Marsland, B.J. \& Kopf, M. CD40-CD4OL cross-talk integrates strong antigenic signals and microbial stimuli to induce development of IL-17-producing CD4 + Tcells. Proc. Natl Acad. Sci. USA 106, 876-881 (2009).

48. Mann, J., Oakley, F., Johnson, P.W. \& Mann, D.A. CD40 induces interleukin-6 gene transcription in dendritic cells: regulation by TRAF2, AP-1, NF-kappa B, AND CBF1. J. Biol. Chem. 277, 17125-17138 (2002).

49. Perona-Wright, G. et al. A pivotal role for CD40-mediated IL-6 production by dendritic cells during IL-17 induction in vivo. J. Immunol. 182, 2808-2815 (2009).

50. Sender, L.Y., Gibbert, K., Suezer, Y., Radeke, H.H., Kalinke, U. \& Waibler, Z. CD40 ligand-triggered human dendritic cells mount interleukin-23 responses that are further enhanced by danger signals. Mol. Immunol. 47, 1255-1261 (2010).

51. Smith, S. et al. Enhanced interferon regulatory factor 3 binding to the interleukin-23p19 promoter correlates with enhanced interleukin-23 expression in systemic lupus erythematosus. Arthritis Rheum. 64, 1601-1609 (2012).

52. Cella, M., Scheidegger, D., Palmer-Lehmann, K., Lane, P., Lanzavecchia, A. \& Alber, G. Ligation of CD40 on dendritic cells triggers production of high levels of interleukin-12 and enhances T cell stimulatory capacity: T-T help via APC activation. J. Exp. Med. 184, 747-752 (1996).

53. Manickasingham, S.P., Edwards, A.D., Schulz, O. \& Reis e Sousa, C. The ability of murine dendritic cell subsets to direct $T$ helper cell differentiation is dependent on microbial signals. Eur. J. Immunol. 33, 101-107 (2003).

54. Tanaka, H., Demeure, C.E., Rubio, M., Delespesse, G. \& Sarfati, M. Human monocyte-derived dendritic cells induce naive $T$ cell differentiation into $T$ helper cell type 2 (Th2) or Th1/Th2 effectors. Role of stimulator/responder ratio. J. Exp. Med. 192, 405-412 (2000).

55. Alcorn, J.F., Crowe, C.R. \& Kolls, J.K. TH17 cells in asthma and COPD. Annu. Rev. Physiol. 72, 495-516 (2010).

56. McAleer, J.P. \& Vella, A.T. Educating CD4 T cells with vaccine adjuvants: lessons from lipopolysaccharide. Trends Immunol. 31, 429-435 (2010).

57. Sakaguchi, S. et al. Essential role of IRF-3 in lipopolysaccharide-induced interferon-beta gene expression and endotoxin shock. Biochem. Biophys. Res. Commun. 306, 860-866 (2003).

58. Lin, K.L., Suzuki, Y., Nakano, H., Ramsburg, E. \& Gunn, M.D. CCR2 + monocyte-derived dendritic cells and exudate macrophages produce influenza-induced pulmonary immune pathology and mortality. J. Immunol. 180, 2562-2572 (2008).

59. Segura, E. et al. Human inflammatory dendritic cells induce Th17 cell differentiation. Immunity 38, 336-348 (2013).

60. Schlitzer, A. et al. IRF4 transcription factor-dependent CD11b + dendritic cells in human and mouse control mucosal IL-17 cytokine responses. Immunity 38, 970-983 (2013).

61. Chen, H. \& Paul, W.E. Cultured NK1.1 + CD4 + T cells produce large amounts of IL-4 and IFN-gamma upon activation by anti-CD3 or CD1. J. Immunol. 159, 2240-2249 (1997).

62. Brandt, E.B., Gibson, A.M., Bass, S., Rydyznski, C. \& Khurana Hershey, G.K. Exacerbation of allergen-induced eczema in TLR4- and TRIFdeficient mice. J. Immunol. 191, 3519-3525 (2013).

63. Guo, B., Chang, E.Y. \& Cheng, G. The type I IFN induction pathway constrains Th17-mediated autoimmune inflammation in mice. J. Clin. Invest. 118, 1680-1690 (2008).

64. Seregin, S.S. et al. TRIF is a critical negative regulator of TLR agonist mediated activation of dendritic cells in vivo. PLOS ONE 6, e22064 (2011).

65. Youn, H.S., Lee, J.Y., Fitzgerald, K.A., Young, H.A., Akira, S. \& Hwang, D.H. Specific inhibition of MyD88-independent signaling pathways of TLR3 and TLR4 by resveratrol: molecular targets are TBK1 and RIP1 in TRIF complex. J. Immunol. 175, 3339-3346 (2005).

66. Lee, J.K., Kim, S.Y., Kim, Y.S., Lee, W.H., Hwang, D.H. \& Lee, J.Y. Suppression of the TRIF-dependent signaling pathway of Toll-like receptors by luteolin. Biochem. Pharmacol. 77, 1391-1400 (2009).

67. Toshchakov, V.U., Basu, S., Fenton, M.J. \& Vogel, S.N. Differential involvement of BB loops of toll-IL-1 resistance (TIR) domain-containing adapter proteins in TLR4- versus TLR2-mediated signal transduction. J. Immunol. 175, 494-500 (2005).

68. Sever, M.L. et al. Cockroach allergen reduction by cockroach control alone in low-income urban homes: a randomized control trial. J. Allergy Clin. Immunol. 120, 849-855 (2007).

69. Hollingsworth, J.W. 2nd et al. The role of Toll-like receptor 4 in environmental airway injury in mice. Am. J. Respir. Crit. Care Med. 170, 126-132 (2004).

70. Nakano, H. et al. Pulmonary CD103(+) dendritic cells prime Th2 responses to inhaled allergens. Mucosal Immunol. 5, 53-65 (2011). 